\title{
Tinmith - Mobile Outdoor Augmented Reality Modelling Demonstration
}

\author{
Wayne Piekarski and Bruce H. Thomas \\ Wearable Computer Laboratory \\ School of Computer and Information Science \\ University of South Australia \\ Mawson Lakes, SA, 5095, Australia \\ wayne@cs.unisa.edu.au,bruce.thomas@unisa.edu.au
}

\begin{abstract}
This paper outlines some of the capabilities of the TinmithMetro modelling system, based on mobile outdoor augmented reality technology. This system implements a new user interface based on tracked pinch gloves and a series of techniques named construction at a distance for the capture and creation of $3 D$ geometry. The user controls the modelling process using hand and head motions, with modelling accuracy guided by the requirements of the user. Using Tinmith-Metro, users are able to model outdoor geometry representing buildings and natural features in an intuitive fashion.
\end{abstract}

\section{Introduction}

We envision mobile outdoor augmented reality (AR) systems being used to provide in situ 3D information displayed to help professionals such as architects, town planners, and engineers. We have been developing applications for mobile AR to give the user the capability to create and visualise their own content in real time. While the capture of existing objects has been performed in the surveying and construction fields, there is also a need for systems that can capture objects from the minds of people for future construction. We believe that AR is an ideal platform to perform both creation and capture of $3 \mathrm{D}$ geometry, since it allows the user to interact with the environment directly, and to verify the results in real time.

These modelling tasks require suitable user interfaces as well as techniques that provide the user with the ability to model and visualise typical objects found outdoors. Our Tinmith-Metro outdoor AR modelling system uses a pair of vision tracked pinch gloves to perform manipulation and interact with a specially designed menu system. We have developed a set of AR techniques, construction at a distance [3], capable of modelling objects that are at a scale larger than the user and at a distance out of arm's reach. Construction at a distance extends previously developed action at a distance concepts by implementing creation as well as the editing of geometry.

\section{User interface}

We have developed an elegant user interface that operates under the poor tracking conditions typically experienced when mobile outdoors. Pinch gloves worn by the user as shown in Figure 1 keep the hands free and provide the ability to enter commands from a menu and perform 3D manipulation. Each finger maps to an item on the menu, and the user can traverse hierarchies and perform actions by pinching the appropriate finger against the thumb. An important feature is that no tracking is required for menu selection, and the user can hold their hands wherever is convenient when not pointing. Using the cursors on the gloves, the user is able to perform translate, rotate, and scale operations, as shown in Figure 3.

Tinmith-Metro is normally operated using an immersive AR display, where the $3 \mathrm{D}$ environment is overlaid onto the view of the physical world. We have also developed external VR interfaces similar to Koller's orbital view (as shown in Figure 2), depicting a virtual landscape and an avatar representing the user. The user's avatar contains a polygon with live video to represent what they are currently viewing. This VR view is useful when the user wishes to gain a higher situational awareness from a bird's eye aerial position.

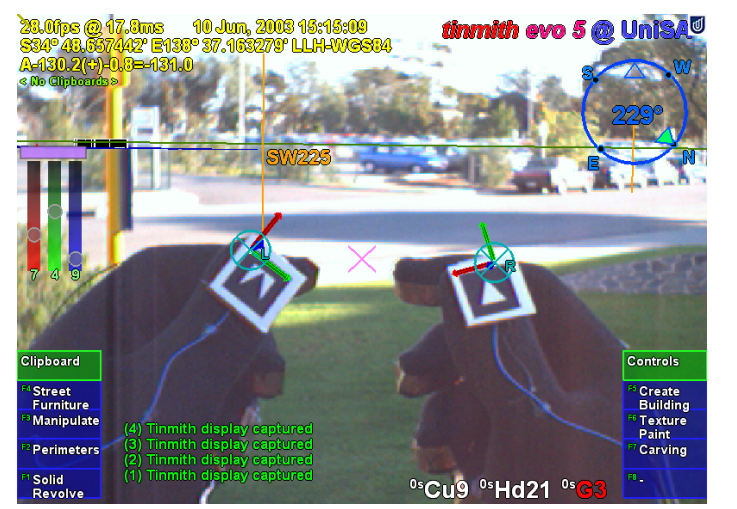

Figure 1 - User interface with tracked gloves and menus

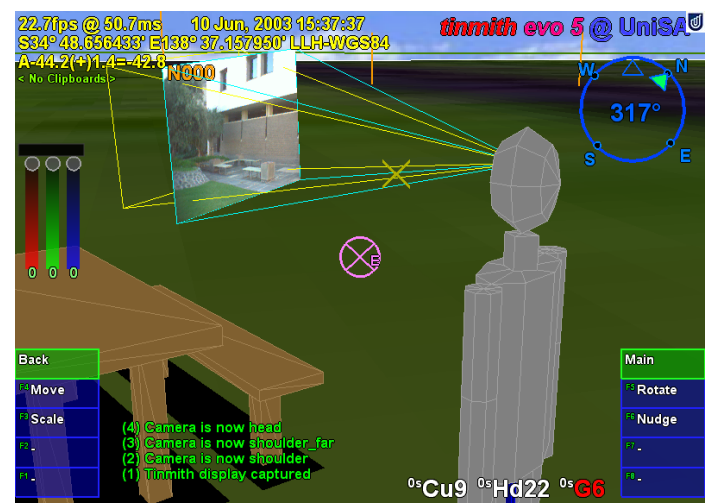

Figure 2 - External VR view of user's avatar with live video 


\section{Construction at a distance}

The previously described construction at a distance concept allows the capture and creation of geometry that is larger than the user, with no prior information or assistance. The use of AR allows users to enter geometry and then verify its accuracy in real time while outdoors. Construction at a distance is based on the use of simple primitives that are combined to build up more complex shapes. Brooks has previously described how using an iterative refinement process guided by the eye is an efficient way of focusing time onto areas of 3D models that require it, capturing the right amount of detail [1]. By iteratively applying modelling operations and the user performing real time comparisons against the physical object, we take advantage of the refinement ideas proposed by Brooks. Construction at a distance is made up of two techniques: body relative and AR working planes, which may be combined in any arbitrary ordering.

\subsection{Body relative techniques}

We use the concept of proprioception [2] to develop geometry capture techniques based solely on the position of the user's body and the orientation of their head. Constructive solid geometry (CSG) is used to create solids of buildings by placing down simple objects such as planes and boxes, and then combining them to produce more interesting shapes. Infinite planes are fired into the world from the user's position and orientation and are used to define the outside surfaces of buildings. The bread crumbs technique is used to place points down, and these may be extruded to create arbitrary concave shapes. The texture map capture technique uses the AR overlay camera to map textures onto existing polygons.

\subsection{AR working planes}

Early CAD systems developed working planes techniques that can be used to specify $3 \mathrm{D}$ points using only $2 \mathrm{D}$ input devices and displays. We have extended these techniques to develop $A R$ working planes that can be fired from the body and then points projected against the surface. The projection carving technique can orthogonally carve away parts of an object from a surface. Projection colouring works similarly but recreates polygons with different colours that are removable to insert surface holes. The surface of revolution technique is used to plot points against an AR working plane and then revolve about an axis to produce round objects such as trees.

\section{Implementation}

The software for Tinmith-Metro is implemented using the Tinmith-evo5 software architecture [4], which is a complete toolkit for the development of high performance 3D virtual environment applications. Our current backpack TinmithEndeavour for performing mobile outdoor AR is shown in Figure 4. This backpack contains a Pentium-III laptop with Nvidia GeForce2 graphics, an InterSense IS-300 orientation sensor, and a Trimble Ag132 GPS for position sensing. The HMD is a Sony Glasstron using Firefly 1394 cameras. Vision tracking of the gloves is implemented using ARToolKit and the head mounted camera, with the fiducial markers mounted onto the thumbs for best visibility, and copper pads used to measure contact between fingers. The backpack is made of polycarbonate and aluminium, and weighs $15 \mathrm{~kg}$ with an operating time of approximately 2 hours.

\section{Conclusion}

In this paper, we have presented a summary of the features available in the Tinmith-Metro mobile outdoor AR modelling application. We have implemented a novel user interface based on tracked pinch gloves and a series of techniques named construction at a distance. Users are able to perform modelling outdoors of objects that are non-existent and beyond arm's reach with real time verification.

\section{References}

[1] Brooks, F. P. What's Real About Virtual Reality? IEEE Computer Graphics and Applications, Vol. 19, No. 6, pp 16-27, 1999.

[2] Mine, M., Brooks, F. P., and Sequin, C. H. Moving Objects In Space: Exploiting Proprioception In Virtual-Environment Interaction. In $A C M$ SIGGRAPH 1997, pp 19-26, Los Angeles, Ca, Aug 1997.

[3] Piekarski, W. and Thomas, B. H. Interactive Augmented Reality Techniques for Construction at a Distance of 3D Geometry. In Immersive Projection Technology / Eurographics Virtual Environments, Zurich, Switzerland, May 2003.

[4] Piekarski, W. and Thomas, B. H. An Object-Oriented Software Architecture for 3D Mixed Reality Applications. In 2nd Int'l Symposium on Mixed and Augmented Reality, Tokyo, Japan, Oct 2003.

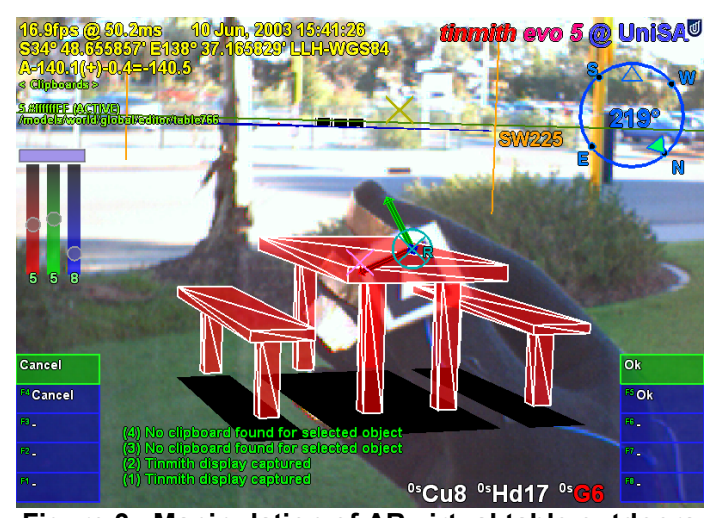

Figure 3 -Manipulation of AR virtual table outdoors
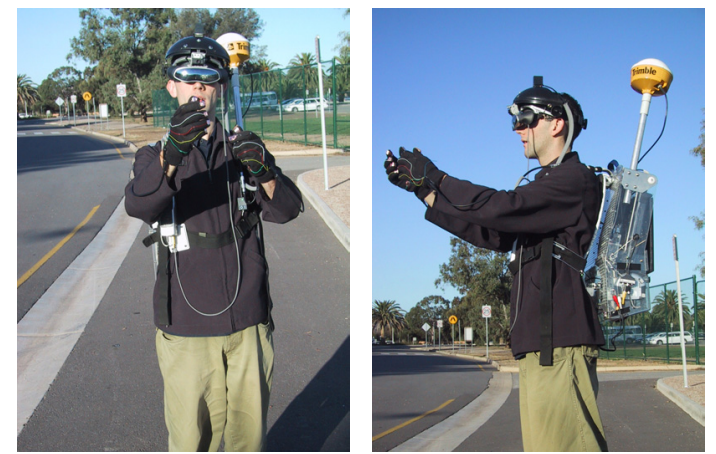

Figure 4 -Tinmith-Endeavour backpack in use outdoors 